\title{
Profils des renversements mortels de machines en milieu agricole au Canada
}

\author{
J. M. DeGroot, M. Sc. (1); C. Isaacs, M. Sc. (1); W. Pickett, Ph. D. (1,2); R. J. Brison, M.H.P. (1,2,3)
}

\section{Résumé}

Introduction : Nous avions pour objectif d'examiner les activités et les circonstances liées aux renversements mortels de machines en milieu agricole.

Méthodologie : Nous avons ciblé les cas de renversements mortels de machines en milieu agricole consignés par le Programme canadien de surveillance des blessures en milieu agricole (PCSBMA) entre 1990 et 2005. Nous avons relevé les renversements de côté et vers l'arrière selon l'âge et le sexe de la victime, l'année, la saison agricole, le type de machine ainsi que l'activité liée à l'accident et le lieu et les circonstances de ce dernier.

Résultats : Le taux de mortalité annuel lié aux renversements au Canada se situait à 9,1 pour 100000 exploitations agricoles. Le nombre de renversements mortels a chuté de $30 \%$ par rapport à la valeur de référence au cours de la période de 16 ans étudiée ( $p=0,004)$. Les renversements mortels ont touché plus souvent les hommes de 50 à 69 ans (renversements de côté) et ceux de 60 à 79 ans (renversements vers l'arrière).

Analyse : Les renversements de côté se produisent lorsque l'opérateur traverse une pente ou roule trop près d'un fossé longeant un chemin ou un champ. Les renversements vers l'arrière se produisent lors de la montée d'une pente, du remorquage d'une machine, du dégagement d'une machine coincée, de l'extraction de souches ou d'arbres et du traînage d'instruments ou de billes de bois. Les programmes de prévention primaire des blessures par renversement devraient cibler ces profils d'accidents.

Mots clés :renversement de machine agricole, blessures en milieu agricole, prévention des blessures, mortalité, structure de protection en cas de renversement, surveillance des blessures

\section{Introduction}

L'agriculture est l'une des industries les plus dangereuses au Canada, avec un taux de mortalité annuel estimé se situant entre 14,6 et 25,6 pour 100000 habitants $^{1}$; elle est tout aussi risquée dans d'autres pays développés ${ }^{2,3}$. Au Canada, les renversements de machine en milieu agricole - qui se produisent lorsqu'un véhicule ou une machine se renverse sur le côté ou bascule vers l'arrière - sont à l'origine d'environ 40 cas
L'efficacité de la structure de protection en cas de renversement (SPR) comme mesure de prévention secondaire permettant de réduire les blessures lors d'un renversement n'est plus à démontrer ${ }^{6-8}$. (On entend par prévention secondaire toute stratégie qui limite la gravité d'une blessure lorsque survient un accident comme un renversement ${ }^{9}$.) Par contre, il existe moins de données sur les stratégies de prévention primaire susceptibles de réduire le nombre de renversements. (On entend par prévention primaire toute stratégie visant à empêcher qu'un accident se produise ${ }^{9}$.)

Plusieurs études portant sur un ensemble d'accidents en milieu agricole ont traité la question des renversements ${ }^{1-4}$. Toutefois, nous n'avons pu trouver aucune étude dans la documentation biomédicale qui décrive les profils courants de survenue des renversements. Le fait de connaître les circonstances entourant ces accidents ainsi que les personnes touchées permettrait de guider les méthodes de prévention primaire et peut-être de mieux cibler les stratégies de prévention secondaire comme l'installation de SPR. En sachant quelles sont les situations les plus dangereuses et les personnes les plus à risque, on pourrait mieux cibler les messages de prévention.

(soit 2,4\% des accide en milieu agricole nécessitant une hospitalisation) et de 21 décès par année (soit $20 \%$ des accidents mortels en milieu agricole) $)^{1,4}$. Les renversements surviennent rapidement, ne laissant pour ainsi dire pas de temps pour effectuer une manœuvre d'évitement; un tracteur peut basculer vers l'arrière jusqu'au point de non retour en 0,75 seconde $^{5}$.
Cette étude a comme objectif l'analyse des activités et des circonstances liées aux renversements mortels en milieu agricole à partir des données d'un programme national de surveillance des blessures en milieu agricole au Canada, et la description des caractéristiques des personnes ayant subi ces accidents.

Rattachement des auteurs

1. Centre de recherche clinique, Hôpital général de Kingston, Kingston (Ontario), Canada

2. Département de santé communautaire et d'épidémiologie, Université Queen's, Kingston (Ontario), Canada

3. Département de médecine d'urgence, Université Queen's, Kingston (Ontario), Canada

Correspondance : Robert Brison, Hôpital général de Kingston, 76, rue Stuart, Kingston (Ontario) K7L 2V7; tél. : 6135482389 ; téléc. : 613 548 1381 ; courriel : brisonr@kgh.kari.net. 


\section{Méthodologie}

\section{Population à l'étude et collecte des données}

Notre étude a été approuvée par le Comité d'éthique de la recherche en sciences de la santé de l’Université Queen's.

La population étudiée inclut toutes les personnes décédées en raison d'un renversement de véhicule ou de machine dans une ferme ou un ranch situé au Canada entre le $1^{\text {er }}$ janvier 1990 et le 31 décembre 2005. Les cas ont été enregistrés par le Programme canadien de surveillance des blessures en milieu agricole (PCSBMA) ${ }^{1}$. Le PCSBMA a relevé les cas d'accidents mortels en milieu agricole dans les bases de données des bureaux des coroners et des médecins légistes en chef des dix provinces canadiennes. À l'aide d'une grille uniformisée, les collaborateurs provinciaux du PCSBMA ont extrait, dans les bureaux mêmes des coroners, les données de chaque dossier ${ }^{1}$. Les renseignements relatifs aux renversements mortels survenus dans la province de Québec en 2004 et en 2005 n'étaient pas disponibles. Le PCSBMA enregistre également les cas d'hospitalisation liés aux accidents en milieu agricole ${ }^{4}$, mais comme notre analyse porte uniquement sur les profils associés aux accidents mortels, nous n'avons pas tenu compte de ces données.

\section{Définition des accidents}

Nous avons étudié les renseignements sur les décès accidentels en milieu agricole et avons codé ceux qui ont été provoqués par un renversement. Selon notre définition, un renversement vers l'arrière se produit lorsqu'un véhicule ou une machine bascule vers l'arrière, les pneus avant tournant autour de l'essieu arrière de $90^{\circ}$ à $180^{\circ}$, tandis que le renversement de côté se produit lorsqu'un véhicule ou une machine bascule sur le côté. Lorsqu'il était impossible de déterminer s'il s'agissait d'un renversement vers l'arrière ou de côté en raison de données insuffisantes, nous avons codé le type de renversement comme non précisé.

\section{Analyse des données}

Nous avons compté le nombre de renversements vers l'arrière et de côté décrits dans le PCSBMA entre 1990 et 2005 . Nous avons établi le profil des renversements de côté et vers l'arrière selon l'âge et le sexe de la

TABLEAU 1

Nombre de renversements mortels en milieu agricole selon le type de renversement, les caractéristiques personnelles de la victime et les circonstances de l'accident

\begin{tabular}{|c|c|c|}
\hline & \multicolumn{2}{|c|}{ Nombre de renversements } \\
\hline & $\begin{array}{c}\text { De côté } \\
(n=221)\end{array}$ & $\begin{array}{l}\text { Vers l'arrière } \\
\quad(n=107)\end{array}$ \\
\hline \multicolumn{3}{|l|}{ Âge de la victime (ans) } \\
\hline 9 et moins & 4 & 3 \\
\hline $10-19$ & 29 & 5 \\
\hline $20-29$ & 13 & 10 \\
\hline $30-39$ & 21 & 7 \\
\hline $40-49$ & 28 & 15 \\
\hline $50-59$ & 35 & 15 \\
\hline $60-69$ & 46 & 25 \\
\hline $70-79$ & 28 & 22 \\
\hline 80 et plus & 17 & 5 \\
\hline \multicolumn{3}{|l|}{ Sexe de la victime } \\
\hline Homme & 207 & 103 \\
\hline Femme & 14 & 4 \\
\hline \multicolumn{3}{|l|}{ Saison agricole } \\
\hline Récolte, juillet-octobre & 126 & 47 \\
\hline Plantation, avril-juin & 46 & 39 \\
\hline Hiver, novembre-mars & 49 & 19 \\
\hline Inconnue & 0 & 2 \\
\hline \multicolumn{3}{|l|}{ Type de machine } \\
\hline Tracteur & 189 & 97 \\
\hline Véhicule hors route & 11 & 8 \\
\hline Engin de chantier & 6 & 2 \\
\hline Véhicule motorisé & 6 & 0 \\
\hline Autre & 9 & 0 \\
\hline \multicolumn{3}{|l|}{ Lieu de l'accident } \\
\hline Champ & 44 & 44 \\
\hline Chemin public & 71 & 6 \\
\hline Chemin de ferme & 39 & 10 \\
\hline Terrain boisé & 14 & 32 \\
\hline Source d'eau & 24 & 3 \\
\hline Cour de ferme & 7 & 4 \\
\hline Voie d'accès & 10 & 0 \\
\hline Bâtiment agricole & 5 & 3 \\
\hline Autre & 4 & 3 \\
\hline Inconnu & 3 & 2 \\
\hline
\end{tabular}

Remarque : Le caractère gras en vert fait ressortir les profils de risque les plus courants.

victime, le type de machine, la saison agricole, le lieu de l'accident, le type d'activité au moment du renversement et la cause la plus probable du renversement. Nous avons calculé les taux annuels globaux et selon l'âge des accidents mortels pour 100000 fermes, puis pour 100000 habitants en utilisant, en guise de dénominateur, les estimations de la population tirées du recensement de l'agriculture de Statistique Canada de $1996^{10}$. Toutes les analyses ont été effectuées à l'aide du logiciel SAS (version 9.2, SAS Institute Inc., Cary, Caroline du Nord, États-Unis). 
FIGURE 1

Nombre de renversements mortels de côté et vers l'arrière en milieu agricole selon l'année, et lignes de tendances

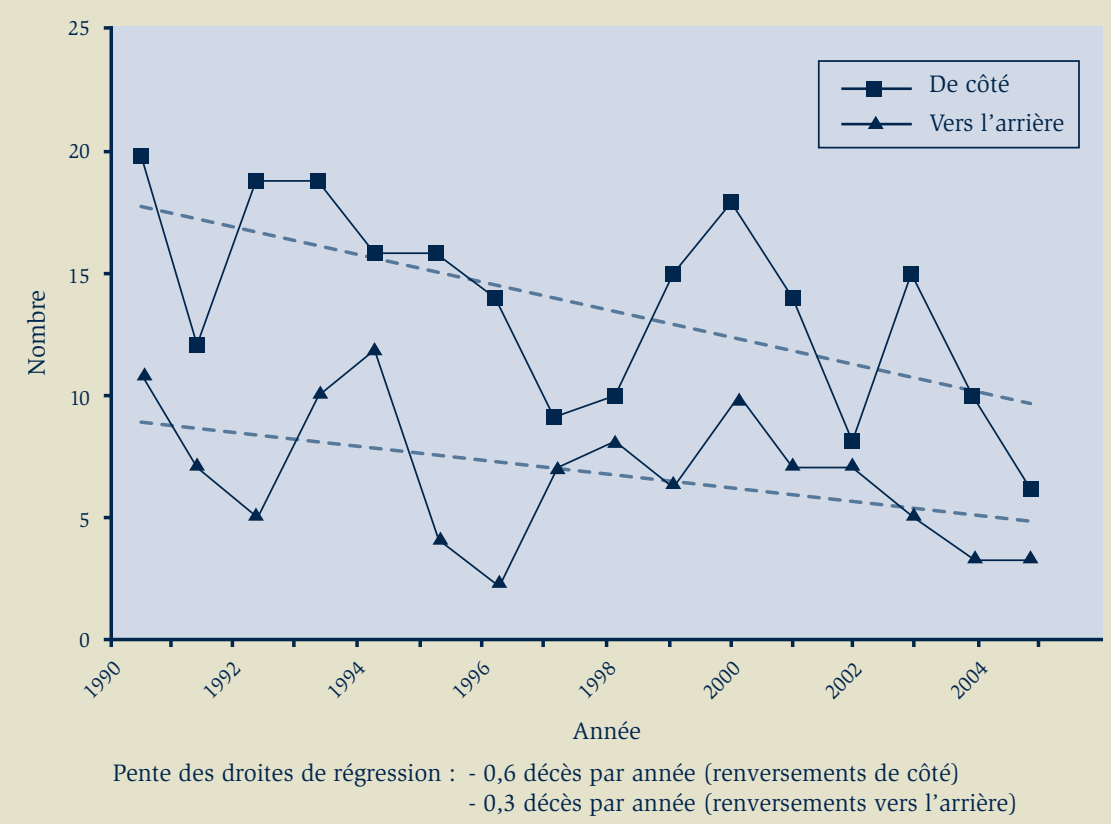

\section{Résultats}

\section{Nombre de renversements}

Sur l'ensemble des accidents mortels en milieu agricole recensés entre 1990 et 2005, soit 1766 décès, 360 (20,4\%) sont attribuables à un renversement. De ces 360 décès, $221(61,4 \%)$ ont été causés par un renversement de côté, 107 (29,7 \%) par un renversement vers l'arrière, et 32 (8,9 \%) par un type de renversement non précisé (tableau 1). Le nombre total de ces accidents a diminué considérablement, passant d'un sommet de 31 renversements en 1990 à un creux de 9 renversements en 2005 $(p=0,004)$, le nombre de renversements de côté et de renversements vers l'arrière variant considérablement d'une année à l'autre. (Remarque : la baisse observée devrait être interprétée avec prudence en raison de l'absence des données provenant du Québec pour les années 2004 et 2005.) Malgré la variabilité du nombre de renversements durant la période étudiée, la tendance à la baisse du nombre de renversements de côté s'est révélée statistiquement significative $(p=0,01)$, la tendance à la baisse du nombre de renversements vers l'arrière demeurant moins claire $(p=0,08)$ (figure 1$)$.

\section{Circonstances des accidents}

Le fait que les groupes plus âgés aient subi un grand nombre de renversements concorde avec la répartition démographique des agriculteurs au Canada ${ }^{10}$. C'est dans le groupe de personnes de 50 à 69 ans que l'on compte le plus grand nombre de décès par renversement de côté, tandis que c'est dans celui des personnes de 60 à 79 ans que l'on observe le plus grand nombre de décès par renversement vers l'arrière (tableau 1). Les renversements mortels touchent plus fréquemment les hommes et les garçons, et se produisent le plus souvent pendant le temps des récoltes et à bord d'un tracteur, et ce, quel que soit le type de renversement. Le deuxième type de machine le plus souvent impliqué dans les renversements de côté et vers l'arrière est le véhicule hors route ( $\mathrm{n}=19$ ); de ce nombre, huit cas de décès (soit 42,1\%) touchaient des enfants de 16 ans et moins. Les rapports d'accident disponibles laissent croire que presque tous ces enfants s'adonnaient à des activités récréatives plutôt qu'à des activités de travail au moment de l'accident. Les renversements de côté se sont produits plus fréquemment dans les champs ou les chemins publics, alors que les renversements vers l'arrière sont survenus plus fréquemment dans un champ ou un terrain boisé (bois, forêt ou verger).

\section{Taux de renversements mortels}

D’après nos estimations, les taux annuels de renversements mortels pour 100000 exploitations agricoles se situent à 9,1 pour l'ensemble des renversements mortels, à 5,6 pour les renversements de côté et à 2,7 pour les renversements vers l'arrière. Les taux annuels de renversements mortels pour 100000 habitants de milieu agricole s'établissent à 2,4 pour l'ensemble des renversements mortels, à 1,6 pour les renversements de côté et à 0,8 pour les renversements vers l'arrière. Les taux annuels selon l'âge augmentent habituellement avec l'âge, tant pour les renversements de côté (minimum : 0,2 pour 100000 chez les enfants de 9 ans et moins; maximum : 13,7 pour 100000 chez les personnes de 80 ans et plus) que pour les 
TABLEAU 2

Activité et facteurs contribuant aux renversements mortels en milieu agricole

\begin{tabular}{|c|c|c|}
\hline & \multicolumn{2}{|c|}{ Nombre de renversements } \\
\hline & $\begin{array}{l}\text { De côté } \\
(\mathrm{n}=221)\end{array}$ & $\begin{array}{l}\text { Vers l'arrière } \\
(\mathrm{n}=107)\end{array}$ \\
\hline \multicolumn{3}{|l|}{ Activité au moment du renversement } \\
\hline Transport & 123 & 8 \\
\hline Travail aux champs & 38 & 23 \\
\hline Foresterie & 14 & 39 \\
\hline Remorquage (extraction) & 7 & 23 \\
\hline Travail dans la cour de ferme & 12 & 9 \\
\hline Fauchage & 12 & 0 \\
\hline Activité récréative & 5 & 2 \\
\hline Entretien des routes & 4 & 1 \\
\hline Inconnue & 6 & 2 \\
\hline \multicolumn{3}{|l|}{ Facteurs contribuant au renversement } \\
\hline $\begin{array}{l}\text { Conduite trop près du bord d'un fossé ou } \\
\text { d'une levée }\end{array}$ & 104 & 12 \\
\hline Conduite dans une pente & 62 & 19 \\
\hline Remorquage (extraction) & 7 & 23 \\
\hline Traînage de billes / d'instruments & 4 & 19 \\
\hline Extraction de souches / d'arbres & 0 & 14 \\
\hline Terrain accidenté & 5 & 7 \\
\hline Chute depuis une rampe & 7 & 1 \\
\hline Collision avec un objet & 6 & 2 \\
\hline Virage & 6 & 1 \\
\hline Transport avec un godet chargé & 6 & 1 \\
\hline Traction de machinerie lourde / d'une remorque & 4 & 3 \\
\hline Bras du tracteur / godet coincé dans la terre & 2 & 0 \\
\hline Inconnu & 8 & 5 \\
\hline
\end{tabular}

Remarque : Le caractère gras en vert fait ressortir les profils de risque les plus courants.

renversements vers l'arrière (minimum : 0,2 pour 100000 chez les enfants de 9 ans et moins; maximum : 4,5 pour 100000 chez les personnes de 70 à 79 ans).

\section{Activités et facteurs contributifs}

Les activités ayant le plus souvent contribué aux renversements de côté sont le transport (en particulier sur les chemins publics) et le travail aux champs. Dans le cas des renversements vers l'arrière, il s'agit le plus souvent d'activités forestières, de travail aux champs et de remorquage ou d'extraction (tableau 2). Les facteurs ayant contribué à la survenue des renversements de côté sont la conduite trop près du bord d'un fossé ou d'une levée et la conduite dans une pente. La plupart des renversements vers l'arrière sont associés 1) à la des profils semblables pour d'autres types d'accidents en milieu agricole ${ }^{2,11-12}$. Le plus grand nombre de décès par renversement de côté et vers l'arrière est observé parmi les adultes de 50 à 79 ans. Cette constatation concorde avec les observations de Myers et collab., qui indiquent que le risque de renversement mortel aux États-Unis augmente avec l'âge. En effet, le taux de décès chez les personnes de 75 ans et plus y était environ 6,5 fois plus élevé que celui des personnes de 25 à 34 ans $^{13}$. Il est reconnu que les agriculteurs ont tendance à travailler au-delà de l'âge normal de la retraite, ce qui est associé à un risque accru d'accident ${ }^{14-17}$. Comme les tracteurs sont faits pour durer longtemps, de nombreux opérateurs âgés utilisent des tracteurs datant d'une époque où les fabricants n'installaient pas systématiquement de $\mathrm{SPR}$, ce qui accroît le risque de décès en cas de renversement ${ }^{18-21}$.

Les renversements de côté se produisent le plus souvent lorsque la machine passe trop près du bord d'une pente raide, habituellement un fossé qui longe un chemin public ou un champ; cela se produit habituellement pendant une activité de transport ou le travail aux champs. Rissanen et Taattola ont établi que les renversements de côté se produisent fréquemment lorsque l'opérateur traverse une pente; il y a alors un déplacement excessif du centre de gravité de la machine, ce qui la fait basculer ${ }^{12}$. La montée d'une pente est aussi une cause fréquente de renversement.

Les renversements vers l'arrière se produisent habituellement dans des circonstances qui relèvent du même mécanisme : remorquage ou extraction de machines, traînage de billes de bois ou d'instruments et extraction de souches ou d'arbres dans un champ ou un terrain boisé. Rissanen et Taattola affirment que les renversements vers l'arrière surviennent principalement lors du remorquage d'un autre tracteur ${ }^{12}$, alors que nos données semblent indiquer que le remorquage, le traînage ou l'extraction de tout objet est la cause la plus fréquente de ce type de renversement. Les renversements vers l'arrière se produisent souvent lorsque l'attelage est inadéquat, c'est-à-dire lorsque l'attache est placée à un niveau plus élevé que l'essieu arrière : environ $60 \%$ des 16 renversements 
vers l'arrière de tracteurs ayant été signalés dans l'État de New York entre 1991 et 1995 impliquaient un attelage inadéquat ${ }^{7}$.

Les rapports sur les renversements insistent souvent sur l'installation de SPR sur les tracteurs comme moyen de réduire la gravité des blessures. Étant donné que la probabilité de décéder à la suite du renversement d'un tracteur équipé d'une SPR est extrêmement faible ${ }^{6-8}$ et que $88 \%$ des renversements mortels en milieu agricole au Canada impliquent un tracteur, on peut supposer qu'un très petit pourcentage des tracteurs visés par notre étude étaient munis d'une SPR, et donc d'une stratégie de prévention secondaire. On estime que l'utilisation d'une SPR et d'une ceinture de sécurité permet de prévenir avec une efficacité de $99 \%$ le décès ou les blessures graves en cas de renversement de tracteur ${ }^{22}$. Les données américaines révèlent une augmentation importante de l'utilisation des SPR, qui est passée de $38 \%$ à $51 \%$ entre 1993 et $2004^{18,23}$.

Puisque les pratiques liées à l'utilisation de la machinerie agricole au Canada reflètent habituellement celles des États-Unis, la baisse des renversements mortels observée tout au long de la période à l'étude est sûrement attribuable à l'utilisation accrue des SPR en milieu agricole au Canada. La diminution des décès que nous avons observée démontre également le bien fondé des stratégies passives de prévention des blessures par des moyens techniques ne nécessitant pas de changement de comportement de la part de l'opérateur. Ces stratégies passives sont efficaces, et leur utilité ne se limite pas à un groupe en particulier, qu'il soit défini par l'âge, le sexe ou le lieu. Les constatations de notre étude font clairement ressortir la nécessité de mettre en œuvre des programmes et des politiques qui encouragent l'application universelle d'innovations passives en matière de sécurité dans le but de protéger les opérateurs de machinerie agricole. En plus de l'utilisation universelle des SPR et des ceintures de sécuritéz ${ }^{2}$, ces innovations pourraient comprendre des dispositifs qui avertissent l'opérateur lorsque la machine se trouve à un angle dangereux, ou qui font en sorte qu'il est très difficile, voire impossible, de placer l'attelage trop haut. Les organisations qui participent à la mise au point et à la promotion de telles innovations sont nombreuses : associations nationales de sécurité (p. ex. l'Association canadienne de sécurité agricole), ministères des gouvernements fédéral, provinciaux et territoriaux (ministère de l'agriculture, ministère du travail et commissions de la sécurité professionnelle et de l'assurance contre les accidents du travail), fabricants de machinerie agricole et organismes connexes, coalitions pour la santé et la sécurité, coroners et médecins légistes.

En plus de s'arrimer aux stratégies de prévention secondaire des accidents telles que les SPR, les programmes de prévention primaire (moins efficaces que les stratégies de prévention secondaire) devraient mettre l'accent sur les causes les plus courantes de renversement et sensibiliser les opérateurs aux dangers connus : pentes latérales et fossés longeant les chemins, (en particulier pendant les périodes de l'année ou le sol est mou), pentes raides, traînage de billes de bois ou d'instruments, remorquage de véhicules et extraction de souches, de billes de bois ou de machines coincées dans un champ.

Le nombre de renversements mortels que nous avons observé chez les enfants de moins de 10 ans fait ressortir la nécessité d'adopter des stratégies de prévention primaire différentes pour ces derniers. Il importe avant tout de restreindre l'accès des jeunes enfants aux lieux de travail agricole associés à des risques connus, comme en témoigne une étude d'une vaste série de $\operatorname{cas}^{24}$. Habituellement, les jeunes enfants n'ont pas les capacités cognitives nécessaires pour reconnaître les situations dangereuses et y réagir de façon appropriée ${ }^{25}$. De plus, il est difficile pour les adultes d'effectuer du travail agricole tout en supervisant de jeunes enfants avec toute la vigilance, la proximité et la constance nécessaires pour les protéger du danger ${ }^{25}$. La seule solution vraiment efficace pour empêcher ces décès par renversement est de laisser les jeunes enfants à l'écart des lieux de travail agricole, et notamment de leur interdire de se trouver à bord ou près d'une machine agricole.

\section{Points forts et limites}

Notre étude se distingue par une analyse détaillée des circonstances des renversements selon le mécanisme en cause. Nous avons utilisé pour cela un ensemble de données solides sur les décès à l'échelle nationale afin de décrire les profils représentatifs des renversements de machine en milieu agricole au Canada. Notre étude comporte aussi quelques limites. Premièrement, nos analyses ont été limitées par les données sur les circonstances des accidents extraites par les collaborateurs provinciaux, qui étaient eux mêmes limités par l'information consignée dans les rapports d'enquête des coroners, les rapports de police et les rapports d'enquête des organismes de santé et de sécurité au travail. Les données sur les victimes et les circonstances des renversements (travailleur à temps plein ou à temps partiel, type de production agricole, présence et utilisation de dispositifs de sécurité comme la SPR ou la ceinture de sécurité) étaient particulièrement limitées. Deuxièmement, comme les données sur les décès au Québec en 2004 et en 2005 n'étaient pas disponibles, le nombre de décès compilés pour ces deux années représente une légère sous-estimation du nombre total prévu à l'échelle nationale. Pour cette raison, le déclin du nombre de renversements mortels observé entre 1990 et 2005 devrait également être interprété avec prudence, bien que les profils d'accidents soient très probablement représentatifs.

\section{Sommaire}

Les renversements de machine constituent l'une des causes les plus fréquentes, bien qu'évitables, de décès accidentel en milieu agricole au Canada. Notre étude a permis de repérer les groupes les plus susceptibles de subir un renversement de côté ou vers l'arrière, et de décrire les circonstances menant le plus souvent à la survenue de ces renversements. Le respect des recommandations sur les SPR et la compréhension des circonstances les plus fréquentes entourant ce type d'accident permettraient d'éviter un nombre important de renversements mortels. 


\section{Remerciements}

Ces travaux ont été financés par l'Association canadienne de sécurité agricole.

Le Programme canadien de surveillance des blessures en milieu agricole (PCSBMA) a été créé en 1995. Des représentants de chacune des dix provinces canadiennes ont été associés aux activités de collecte des données sur lesquelles repose cette étude. Depuis 2010, ce programme a un nouveau nom : Surveillance des blessures dans le secteur agricole au Canada (SBAC). Il est géré par le Alberta Centre for Injury Control and Research. SBAC est financé par une subvention de l'Association canadienne de sécurité agricole et par Agriculture et Agroalimentaire Canada. Cependant, ces organismes n’ont participé ni à la conception de l'étude, ni à la collecte, l'analyse ou l'interprétation des données, ni à la rédaction du document, ni à la décision de soumettre cet article pour publication.

\section{Références}

1. Pickett W, Hartling L, Brison RJ, Guernsey JR. Fatal work-related farm injuries in Canada, 1991-1995. Canadian Agricultural Injury Surveillance Program. CMAJ. 1999;160:1843-8.

2. Franklin RC, Mitchell RJ, Driscoll TR, Fragar LJ. Agricultural work-related fatalities in Australia, 1989-1992. J Agric Saf Health. 2001; 7:213-27.

3. Hard DL, Myers JR, Gerberich SG. Traumatic injuries in agriculture. J Agric Saf Health. 2002;8:51-65.

4. Pickett W, Hartling L, Dimich-Ward H, Guernsey JR, Hagel L, Voaklander DC, et al. Surveillance of hospitalized farm injuries in Canada. Inj Prev. 2001;7:123-28.

5. Hathaway LR, Riney LA, editors. Fundamentals of machine operation: agricultural safety. Moline (IL): Deere \& Company Service Training; 1987.

6. Centers for Disease Control and Prevention. Public health focus: effectiveness of rollover protective structures for preventing injuries associated with agricultural tractors. MMWR. 1993;42:57-9.
7. Centers for Disease Control and Prevention. Fatalities associated with improper hitching to farm tractors-New York, 1991-1995. MMWR. 1996;45:307-11.

8. Springfeldt B, Thorson J, Lee BC. Sweden's thirty-year experience with tractor rollovers. J Agric Saf Health. 1998;4:173-80.

9. Conroy C, Fowler J. The Haddon matrix: applying an epidemiologic research tool as a framework for death investigation. Am J Forensic Med Pathol. 2000;21:339-42.

10. Statistique Canada. Recensement de l'agriculture 2006, [Internet], Ottawa (Ontario), Statistique Canada; [modification le 5 oct. 2009; consultation le 30 déc. 2010]. Consultable en ligne à la page : http:// www.statcan.gc.ca/ca-ra2006/index-fra.htm

11. Locker AR, Pickett W, Hartling L, Dorland JL. Agricultural machinery injuries in Ontario, 1985-1996: a comparison of males and females. J Agric Saf Health. 2002;8:215-23.

12. Rissanen P, Taattola K. Fatal injuries in Finnish agriculture, 1988-2000. J Agric Saf Health. 2003;9:319-26.

13. Myers JR, Hendricks KJ. Agricultural tractor overturn deaths: assessment of trends and risk factors. Am J Ind Med. 2009;53:662-72.

14. Horsburgh S, Feyer A-M, Langley JD. Fatal work related injuries in agricultural production and services to agriculture sectors of New Zealand, 1985-94. Occup Environ Med. 2001;58:489-95.

15. Myers JR, Hard DL. Work-related fatalities in the agricultural production and services sectors, 1980-1989. Am J Ind Med. 1995;27:51-63.

16. Soloman C. Accidental injuries in agriculture in the UK. Occup Med. 2002;52:461-6.

17. Voaklander DC, Hartling L, Pickett W, Dimich-Ward H, Brison RJ. Work-related mortality among older farmers in Canada. Can Fam Physician. 1999;45:2903-10.

18. Loringer KA, Myers JR. Tracking the prevalence of rollover protective structures on
U.S. farm tractors: 1993, 2001, and 2004. J Safety Res. 2008;39:509-17.

19. May JJ, Sorensen JA, Burdick PA, EarleRichardson GB, Jenkins PL. Rollover protection on New York tractors and farmers' readiness for change. J Agric Saf Health. 2006;12:199-213.

20. Sanderson WT, Madsen MD, Rautiainen R, Kelly KM, Zwerling C, Taylor CD, et al. Tractor overturn concerns in Iowa: perspectives from the Keokuk county rural health study. J Agric Saf Health. 2006;12:71-81.

21. Wilkins JR III, Engelhardt HL, Bean TL, Byers MV, Crawford JM. Prevalence of ROPSequipped tractors and farm/farmer characteristics. J Agric Saf Health. 2003;9:107-18.

22. Murphy DJ, Buckmaster DR. Rollover protection for farm tractor operators [Internet]. Pennsylvania State University (PA): Agricultural and Biological Engineering; 2003 [consultation le 14 décembre 2010]. PDF téléchargeable à partir du lien : http:// www.abe.psu.edu/extension/factsheets/e/ E42.pdf

23. Myers JR, Snyder K. Roll-over protective structure use and cost of retrofitting tractors in the United States, 1993. J Agric Saf Health. 1995;1:185-97.

24. Brison RJ, Pickett W, Berg R, Linneman J, Zentner J, Marlenga B. Fatal agricultural injuries in preschool children: risks, injury patterns and strategies for prevention. CMAJ. 2006;174:1723-6.

25. Morrongiello B, Pickett W, Berg R, Linneman J, Brison RJ, Marlenga BL. Adult supervision and pediatric injuries in the agricultural worksite. Accid Anal Prev. 2008;40:1149-56 